\title{
PROJETO DE MINIMIZAÇÃO DE RESÍDUOS SÓLIDOS NO RESTAURANTE CENTRAL DO CAMPUS DE SÃO CARLOS DA UNIVERSIDADE DE SÃO PAULO
}

\author{
Raquel de Luca Menezes \\ Departamento de Engenharia de Engenharia de Produção - Escola de Engenharia de São Carlos- \\ USP \\ Av. Trabalhador São-carlense, 400 - Cep 13566-590 - São Carlos - SP \\ Fernando César Almada Santos \\ Departamento de Engenharia de Engenharia de Produção - Escola de Engenharia de São Carlos- \\ USP \\ Av. Trabalhador São-carlense, 400 - Cep 13566-590 - São Carlos - SP \\ Patrícia Cristina Silva Leme \\ Programa USP Recicla \\ Av. Trabalhador São-carlense, 400 - Cep 13566-590 - São Carlos - SP
}

\begin{abstract}
This paper presents the project for the minimisation of the solid residues which are produced by the Central Restaurant of the São Carlos Campus of the University of São Paulo which was carried out by a partnership involving the USP Recicla Programme and the Department of Production Engineering. The basis of this programme is the $3 R$ 's principle: Reducing, Reusing and Recycling. There was also a great concern with the involvement of the users' community of this restaurant. The cost reduction originated from the implementation of $3 R$ 's measures was also estimated. The scheduling of this project consisted of the following phases: the data collection, the diagnosis of garbage, the proposition of measures for the residues minimisation and the implementation of the appropriate measures, after a choice involving economic, hygienic and personnel criteria. There will not be a definite conclusion of this project, on the contrary, it continues to be practised in the Central Restaurant. It is believed that this project contributed for the implementation of actions related to the knowledge of the solid residue area.
\end{abstract}

key words: minimisation of solid residues, garbage diagnosis, planning and implementation .

\section{INTRODUÇÃO}

A dinamização produtiva e a desigualdade sócio-econômica são duas grandes características do mundo contemporâneo. Nota-se, num mesmo cenário, marcas de avanço da tecnologia e necessidades básicas da humanidade ainda não satisfeitas.

Na sociedade urbano-industrial criou-se um padrão onde é necessário consumir mais e mais para sustentar a fonte dos capitais, enquanto a maioria pobre é cada vez mais marginalizada nesse sistema. Esses fatores agravam a questão ambiental, visto que o crescimento das necessidades de matérias-primas e energia, e a produção e consumo cada 
vez maiores esbarram nos limitados recursos do planeta. Nós, enquanto humanidade, colocamos em risco ecossistemas, somos responsáveis por parte da diminuição da biodiversidade, ameaçando a nossa própria sobrevivência (ESTADO DE SÃO PAULO, $\mathrm{s} / \mathrm{d})$.

Visando a proteção e a melhoria do meio ambiente elaborou-se, durante a Conferência das Nações Unidas sobre Meio Ambiente e Desenvolvimento - RIO 92 - uma Agenda, assinada por 177 países, com diretrizes para o século 21. Nesta chamada Agenda 21, lê-se que "as principais causas da deterioração ininterrupta do meio ambiente mundial são os padrões insustentáveis de consumo e produção, especialmente nos países industrializados. Motivo de séria preocupação, tais padrões de consumo e produção provocam o agravamento da pobreza e dos desequilíbrios" (ESTADO DE SÃO PAULO, $\mathrm{s} / \mathrm{d}$ ). Assim, torna-se indispensável o desenvolvimento de políticas e estratégias para estimular mudanças nos padrões de consumo e produção.

No capítulo 4 da mesma Agenda 21, afirma-se que a qualidade do meio ambiente de nosso planeta e um desenvolvimento sustentável e ambientalmente saudável só poderá ser alcançado através de mudanças nas atitudes tomadas em relação aos resíduos da sociedade (ESTADO DE SÃO PAULO, s/d). São citadas as seguintes mudanças: "redução ao mínimo dos resíduos; aumento ao máximo da reutilização e reciclagem ambientalmente saudáveis dos resíduos; promoção e tratamento ambientalmente saudáveis dos resíduos; ampliação dos serviços que se ocupam dos resíduos". Promulga-se que "a sociedade precisa desenvolver formas eficazes (...) para reduzir a geração de resíduos e de produtos descartados" (ESTADO DE SÃO PAULO, s/d)

Assim, o lixo, enquanto resíduo sólido, é de responsabilidade de todos e cada um desempenha papel importante na mudança da realidade ambiental, de acordo com sua função na sociedade (CALDERONI, 1997; CASCINO, JACOBI \& OLIVEIRA, 1998; CONSUMERS INTERNATIONAL, 1998; JARDIM, 1995).

\section{O PROGRAMA INSTITUCIONAL USP RECICLA}

A Universidade assume grande papel na formação de cidadãos críticos, instrumentados para a implementação dessas necessárias mudanças. Como promotora do desenvolvimento de novas tecnologias pode, ainda, estabelecer diretrizes e incentivar pesquisas sobre os diversos aspectos do controle da poluição relacionada com os resíduos. Além disso, a universidade desempenha importante papel como divulgadora e estimuladora de novas idéias, convidando a população a se empenhar na busca de soluções para a problemática dos resíduos.

A Universidade de São Paulo, diante das discussões levantadas na Conferência Rio92 e preocupada com seus próprios resíduos sólidos, criou, em 1994, o programa institucional USP Recicla - da Pedagogia à Tecnologia (BLAUTH e LEME, 1998).

O programa USP Recicla tem como principal objetivo desenvolver na comunidade universitária uma mentalidade voltada à preservação ambiental. Baseado no princípio dos “3Rs" (Redução no consumo e desperdício, Reutilização e Reciclagem), atua como um programa educativo de minimização de resíduos.

Através de diagnósticos do lixo e observação dos processos operacionais nas Unidades (termo genérico que abrange faculdades, museus ou institutos) que compõem os 
seus 6 campi, são oferecidas diretrizes para implementação do projeto. Com esta atuação, a USP já conseguiu a redução de cerca de $50 \%$ em peso do lixo universitário.

O Restaurante Universitário é uma das unidades do Campus de São Carlos que não possui o programa USP Recicla implantado e oferece cerca de 1500 refeições por dia. Como um dos "resultados" desta atividade, tem-se a geração de grande quantidade de resíduos sólidos, provenientes tanto na preparação dos alimentos, como do resto das bandejas dos usuários. Esses resíduos (restos de alimentos, copos plásticos, guardanapos, latas etc) têm como destino final o aterro municipal de São Carlos.

Torna-se, assim, indispensável a implantação de um projeto de minimização de resíduos. Além disso, por possuir um grande fluxo populacional, o Restaurante Universitário é um ótimo local para divulgação de um projeto desta natureza, fazendo com que a comunidade universitária que freqüenta o Restaurante, sinta-se mais envolvida com a problemática do lixo.

O período de realização da primeira edição deste projeto foi de março a dezembro de 2000, lembrando-se que suas atividades de planejamento e execução encontram-se em continuidade até o momento atual.

Os levantamentos e planos de ação desenvolvidos no decorrer deste projeto tiveram apoio financeiro da Fundação para o Incremento à Pesquisa e do Aperfeiçoamento Industrial - FIPAI.

\section{OBJETIVOS PROJETO DE MINIMIZAÇÃO DE RESÍDUOS SÓLIDOS}

Os objetivos Projeto de Minimização de resíduos sólidos são:

- implantação de um projeto de minimização de resíduos no Restaurante Universitário, incluindo a redução do consumo e do desperdício, a reutilização e, como última opção, a reciclagem;

- desenvolver, junto à comunidade usuária e funcionários do Restaurante Universitário, uma mentalidade voltada para a preservação ambiental;

- estimar os custos evitados com a implantação de medidas de combate ao desperdício, reutilização e reciclagem de materiais.

\section{ETAPAS DO PROJETO}

Este projeto realiza-se por meio de parceria entre o Departamento de Engenharia de Produção e Comissão do Programa USP Recicla do Campus de São Carlos. Esta comissão é constituída por um representante docente de cada uma das unidades e duas educadoras ambientais.

Partindo do princípio dos "3Rs", a implantação do projeto no Restaurante consta das seguintes etapas:

- levantamentos iniciais; 
- construção de um plano de minimização de resíduos;

- trabalho educativo;

- assistência na implementação das mudanças;

- divulgação dos resultados e feedback

Nos levantamentos iniciais, realiza-se o preenchimento de uma planilha referente aos processos operacionais da unidade, objetivando obter informações como sistema utilizado para coleta do lixo, destinação deste e gastos com materiais descartáveis. Além disto, elabora-se um diagnóstico do lixo da unidade, constituído por três amostras do lixo gerado em 24 horas. Os resíduos gerados serão analisados qualitativa e quantitativamente.

Para a construção de um plano de minimização de resíduos, realiza-se um estudo baseado no diagnóstico do lixo e na observação dos meios operacionais do Restaurante, incluindo a seção de alimentação e administrativa. Determinam-se também diretrizes visando a minimização dos resíduos sólidos.

No que se refere ao trabalho educativo, promovem-se encontros educativos com os funcionários do Restaurante orientados pela educadora do USP Recicla, visando estimular os servidores a implementarem ações que impliquem o menor desperdício e geração de resíduos. Constrói-se um mural no refeitório, informando aos freqüentadores os passos da implementação do projeto de minimização no Restaurante. Desta forma, convida-se a comunidade universitária a também se empenhar na melhoria ambiental, através de mudanças de hábitos com relação à geração de resíduos.

Busca-se ainda a assistência na implementação das mudanças, por meio do trabalho em parceria com os funcionários da unidade para a efetivação e aprimoramento das diretrizes definidas na etapa de construção de um plano de minimização de ruídos.

Por fim, divulgação dos resultados do Projeto Minimização de Resíduos por meio da atualização das seguintes informações do mural do USP Recicla: os passos da implementação do projeto, os objetivos, as atitudes e os resultados. O objetivo desta divulgação é obter feedback por parte da comunidade.

O Projeto Minimização de Resíduos mantém-se através de re-diagnósticos periódicos do lixo para avaliar a evolução do projeto no restaurante, como a economia referente à redução dos custos e desperdício de materiais no orçamento da unidade.

\section{DIAGNÓSTICO DO LIXO}

O diagnóstico do lixo baseia-se em análise qualitativa e quantitativa de amostras do lixo gerado no Restaurante Universitário. A fim de obter um resultado mais representativo da situação do lixo gerado por essa Unidade, foram analisadas três amostras (cada uma referente ao lixo gerado em dois dias) em épocas diferentes do ano, levando-se em conta a sazonalidade do consumo e o cardápio do dia, uma vez que estes itens alteram o resultado do diagnóstico. 
Deste modo, observou-se que o lixo gerado pelo Restaurante Central do Campus Universitário de São Carlos da USP de, referente ao preparo e distribuição de dois almoços e um jantar (cerca de 2000 bandejas) tem, em média, $360 \mathrm{~kg}$ e ocupa um volume de 4.973 litros. Segue a Tabela 1 com os principais itens encontrados no lixo da unidade e seus respectivos volumes e massas.

TABELA 1 - Diagnóstico Médio do Restaurante Universitário (gerado em 2 dias)

\begin{tabular}{|c|c|c|c|c|c|}
\hline & Material & $\begin{array}{r}\text { Peso } \\
(\mathrm{kg})\end{array}$ & $\begin{array}{l}\text { Peso } \\
(\%)\end{array}$ & $\begin{array}{c}\begin{array}{c}\text { Volume } \\
\text { (litros) }\end{array} \\
\end{array}$ & $\begin{array}{c}\text { Volume } \\
(\%)\end{array}$ \\
\hline & orgânicos & 268,6 & 74,6 & 1989,3 & 40,0 \\
\hline \multirow{3}{*}{ PAPEL } & papel & 9,7 & 2,7 & 323,3 & 6,5 \\
\hline & papelão & 4,0 & 1,1 & 64,7 & 1,3 \\
\hline & papel banheiro & 5,8 & 1,6 & 189,0 & 3,8 \\
\hline \multirow[t]{5}{*}{$\begin{array}{l}\text { PLÁS- } \\
\text { TICOS }\end{array}$} & $\begin{array}{l}\text { copos plásticos } \\
\text { sacos plásticos } \\
\text { guardanapos }\end{array}$ & 54,0 & 15,0 & 1477,0 & 29,7 \\
\hline & vinagre (plástico) & 1,4 & 0,4 & 44,7 & 0,9 \\
\hline & plástico & 12,2 & 3,4 & 770,8 & 15,5 \\
\hline & metal & 4,3 & 1,2 & 114,4 & 2,3 \\
\hline & TOTAL & 360,0 & 100,0 & 4973,2 & 100,0 \\
\hline
\end{tabular}

\section{PROPOSTAS DE MEDIDAS PARA A MINIMIZAÇÃO DE RESÍDUOS}

Visando a construção do processo de transformação, foi proposta e aprovada a criação de uma comissão formada por funcionários do Restaurante, responsável pelo gerenciamento de medidas voltadas à minimização de resíduos. Essa comissão se reunia periodicamente com a equipe do USP Recicla para discussão e implementação do projeto.

Após a etapa de levantamento de dados, foram sugeridas algumas medidas para minimização de resíduos sólidos no Restaurante Central (Tabela 2). Estas medidas foram apresentadas e discutidas com a comissão, que avaliou sua viabilidade. 
TABELA 2 - Sugestões de medidas para minimização de resíduos sólidos no Restaurante Universitário e respectivos impactos na geração do lixo

\begin{tabular}{|c|c|c|c|}
\hline \multirow[t]{2}{*}{ MATERIAL / MEDIDA } & \multicolumn{3}{|c|}{ IMPACTO NO LIXO / MÊS } \\
\hline & $\begin{array}{l}\text { Volume } \\
\text { (litros) }\end{array}$ & $\begin{array}{c}\text { Peso } \\
(\text { kg) }\end{array}$ & Unidades \\
\hline $\begin{array}{l}\text { Copos plásticos: } \\
1-) \text { substituição por copos duráveis. }\end{array}$ & & & -56000 \\
\hline 2-) limitar a utilização. & \multirow{3}{*}{-9800} & \multirow{3}{*}{$-378,0$} & -28000 \\
\hline $\begin{array}{l}\text { Guardanapos: } \\
\text { 1-) reduzir a utilização. }\end{array}$ & & & -14000 \\
\hline $\begin{array}{l}\text { Sacos plásticos para talher: } \\
\text { 1-) abolir. }\end{array}$ & & & -28000 \\
\hline $\begin{array}{l}\text { Frascos de detergente: } \\
\text { 1-) comprar a granel com } \\
\text { reuso do frasco. } \\
\text { 2-)substituição por sabão em pedra } \\
\text { feito com óleo usado. }\end{array}$ & -140 & $-21,0$ & -182 \\
\hline
\end{tabular}

\section{Orgânico (1):}

1-) doação para criadores de animais.

Frasco plástico de vinagre:

1-) reutilização com embalagem de vidro.

\begin{tabular}{|c|c|c|c|}
\hline \multirow{2}{*}{-27860} & $-3760,4$ & $*$ & $\mathbf{R E}$ \\
$\mathbf{U}$ \\
$\mathbf{T I}$ \\
-630 & $-22,4 \mathrm{~kg}$ & -378 & $\begin{array}{c}\mathbf{L I} \\
\mathbf{Z A} \\
\mathbf{R}\end{array}$ \\
\cline { 2 - 5 }
\end{tabular}

\section{Orgânico ( 1 ): *}

1-) compostagem.

\section{Papelão:}

1-) coleta seletiva.

Papel (de escritório e em

1-) coleta seletiva.

Orgânico ( 2 ): **

1-) compostagem.

doação para criadores de porcos, com

esterilização.

* proveniente do pré-preparo e açougue.

** proveniente das sobras das bandejas. 


\section{REALIZAÇÃO DE MEDIDAS PARA A MINIMIZAÇÃO DE RESÍDUOS}

A avaliação das medidas propostas e a criação de outras foram baseados na disponibilização de recursos humanos e financeiros e em aspectos higiênicos e operacionais envolvidos nas atividades realizadas pela unidade.

Focalizando a redução do lixo orgânico, proveniente do pré-preparo, açougue e sobras das bandejas, foram feitas duas sugestões: encaminhamento para criadores de animais e criação de uma composteira (PEREIRA NETO, 1996). A primeira apresentou problemas sanitários relacionados à armazenagem desse lixo até seu destino final. A segunda foi excluída por falta de recursos. A solução encontrada foi a realização de trabalhos educativos junto à comunidade usuária e funcionários visando a redução do desperdício.

Para o excesso de copos plásticos presentes no lixo gerado pelo Restaurante Central, escolheu-se a limitação de seu uso, uma vez que sua substituição por copos duráveis geraria problemas operacionais relacionados a sua higienização. Esta medida, associada a um trabalho educativo através de murais informativos, não encontrou resistência por parte dos usuários e representou uma diminuição de $50 \%$ da quantidade deste componente no lixo total gerado pela unidade.

Um novo sistema de distribuição de talheres sem a necessidade do uso de sacos plásticos foi adotado, reduzindo em $100 \%$ a quantidade deste item no total de resíduos gerados. Além disto, este novo sistema desvinculou a distribuição de guardanapos à de talheres, possibilitando a escolha de uma método que atende a demandas individuais por este item, diminuindo seu desaproveitamento.

O papelão e papel de escritório passaram a ser destinados à coleta seletiva.

Os frascos de detergente e vinagre não foram substituídos por questões operacionais.

Um aspecto muito importante para a implementação das medidas foi a sensibilização dos funcionários e usuários quanto à questão ambiental, através de murais informativos. A comissão de funcionários do Restaurante também foi imprescindível nesse processo, uma vez que possibilitou maior coerência na escolha e priorização das ações adotadas.

A adoção dessas mediadas gerou indiretamente otimização em alguns processos do Restaurante Central, possibilitando uma maior diversificação dos cardápios oferecidos e, consequentemente, aumentando o número total de refeições servidas pela unidade.

\section{CONSIDERAÇÕES FINAIS}

Por se tratar de um trabalho de caráter educativo, a redução dos resíduos sólidos do Restaurante Universitário exige a conscientização de seus usuários e funcionários quanto à questão ambiental, para que assim o projeto possa permanecer em contínuo desenvolvimento.

Todas as fases do projeto, com exceção dos levantamentos iniciais, devem ser retomadas e reavaliadas periodicamente. Desta forma, estabelece-se o caráter contínuo do projeto. 
Avalia-se positivamente o desenvolvimento do Projeto de Minimização de Resíduos Sólidos no Restaurante Central do Campus de São Carlos da Universidade de São Paulo, uma vez que este proporcionou tanto o aprofundamento de conhecimentos relativos aos dos resíduos sólidos, como a contribuição ativa para sua solução dentro de uma Unidade do campus de São Carlos da USP.

\section{BIBLIOGRAFIA}

BLAUTH, P. , LEME, P.C.S. USP Recicla. In: EIGENHEER, E.M., org. Coleta seletiva de lixo: experiências brasileiras. Rio de Janeiro, UFF/CIRS/Ecomarapendi, 1993.

CALDERONI, S. O\$ bilhõe\$ perdido\$ no lixo. São Paulo, Humanitas, 1997.

CASCINO,F., JACOBI, P., OLIVEIRA, J., orgs. Educação, Meio Ambiente e Cidadania. São Paulo, Secretaria de Estado do Meio Ambiente, 1998.

CONSUMERS INTERNATIONAL. Consumo sustentável. São Paulo, Secretaria de Meio Ambiente, 1998.

EIGENHEER, E.M., org. Coleta seletiva de lixo: experiências brasileiras. Rio de Janeiro, UFF/CIRS/Ecomarapendi, 1993.

JARDIM, N. S. et al. Lixo municipal: manual de gerenciamento integrado. São Paulo, IPT - CEMPRE, 1995.

ESTADO DE SÃO PAULO. Resíduos sólidos: agenda 21, projetos de lei federal e estadual. São Paulo, Secretaria de Meio Ambiente, s. d.

ESTADO DE SÃO PAULO. Guia pedagógico do lixo. São Paulo, Secretaria de Meio Ambiente, 1998a

ESTADO DE SÃO PAULO. Resíduos sólidos: um problema global. São Paulo, Secretaria de Meio Ambiente, 1998b.

PEREIRA NETO, J.T. Manual de compostagem. Belo Horizonte, UNICEF, 1996. 